\title{
Nanoindentation technique as a probe for characteristic deformation size
}

\author{
Jinxu Li ${ }^{1}{ }^{2}$, Takahito Ohmura ${ }^{1}$, Mitsuhiro Murayama ${ }^{1}$, Toru Hara ${ }^{1}$ and Kaneaki Tsuzaki ${ }^{1}$ \\ 1. Steel Research Center, National Institute for Materials Science, Tsukuba, Japan \\ 2. Department of Materials Physics, University of Science \& Technology Beijing, Beijing 100083, China
}

\begin{abstract}
A system of nanoindentation combined with atomic force microscopy was applied to a multi-phase material for evaluating its mechanical property. A tempered martensitic steel (SCM440) with a tensile strength of $1600 \mathrm{MPa}$ was tested as an example of multiphase materials, and three types of tungsten single crystals, with respective (100), (110) and (111) surface, were also tested as reference. In the tempered martensitic steel, the load-displacement behavior depends on measured positions at the specimen surface and/or sizes of deformation zone. This behavior represents inhomogeneity of the multiphase microstructure where each component has a different mechanical property and strength. When the contact depth reaches $60 \mathrm{~nm}$ depth, the standard deviation of nanohardness becomes a constant value. Therefore, $60 \mathrm{~nm}$ could be the critical contact depth for disappearance of inhomogeneity effect on the deformation behavior. The constitution of the standard deviation of hardness was discussed in some detail.
\end{abstract}

\section{Key words: Nanoindentation, Deformation zone, Tempered martensitic steel, Tungsten single crystal}

\section{INTRODUCTION}

A single phase can greatly influence the mechanical behavior of a bulk multi-phase material. The integral mechanical property of the material originates from the mechanical properties of all component phases and their distribution and interaction. Therefore, the evaluation of the mechanical property of each single phase, including very fine microstructure, is essential for understanding and controlling mechanical behavior of the bulk multi-phase material.

Advances in testing methods have led to general availability of determining the mechanical properties of materials in small volumes. Nowadays, the measurement of the mechanical properties of materials at the nanometer scale has been pursued by both the nanomechanics and the atomic force microscopy (AFM) communities. One of the advanced technique is a system of nanoindentation combined with AFM. The technique allows in-situ imaging of a sample surface with a diamond indenter tip, and makes it possible to obtain microstructural information of the exact location where the indenter is performed. Nanoindenters provide accurate measurements of the continuous variation of indentation load (P) down to the order of micro-Newtons as a function of the indenter penetration depth (h) down to the order of nanometers. Researches on nanoindentation have been performed on a variety of materials [1-4] with the objective of extracting mechanical properties, such as elastic moduli $[1,5,6]$, hardness [7, 8] and time-dependent deformation effects [9]. On the other side, the integral mechanical property of the material originates from the mechanical properties of all component phases and their distribution and

Corresponding author:

Jinxu Li, LI.Jinxu@nims.go.jp interactions.

In order to demonstrate the performance of the integrated nanoindentation system, a tempered martensitic steel (SCM440) with a tensile strength of $1600 \mathrm{MPa}$ was tested as an example of multi-phase materials, and three types of ungsten single crystals, which each surface is parallel to one of bcc low index planes, i.e., (100), (110) and (111), were also tested as reference. According to the former investigations, the microstructure of carbon lath martensite consists of four structural units; lath, block, packet and prior austenite grain [10-12]. Thus it is expected that the loaddisplacement behavior of the tempered martensitic steel depends on measured positions and/or sizes of deformation zone corresponding to magnitude of the applied peak load. This behavior represents inhomogeneity of the multi-phase microstructure where each component has different mechanical property. We also discuss the relationship between microstructural features and standard deviation distribution of nanoindentation data in order to determine the critical condition for disappearance of inhomogeneity effect on the deformation behavior, which is important to materials design for MEMS/MST devices.

\section{EXPERIMENTAL}

The chemical composition of the steel was $0.40 \% \mathrm{C}$, $0.25 \% \mathrm{Si}, \quad 0.79 \% \mathrm{Mn}, \quad 0.016 \% \mathrm{P}, \quad 0.017 \% \mathrm{~S}, \quad 1.12 \% \mathrm{Cr}$, $0.17 \% \mathrm{Mo}, 0.02 \% \mathrm{Al}, 0.002 \% \mathrm{Ti}, 0.0008 \% \mathrm{O}, 0.0037 \% \mathrm{~N}$, and the balance $\mathrm{Fe}$ (mass\%). A bar with $7.75 \mathrm{~mm}$ diameter was austenitized at $1153 \mathrm{~K}$ for 900 s in argon gas atmosphere and quenched by water spray, resulting in a prior austenite grain size of about $24 \mu \mathrm{m}$. It was then tempered at $673 \mathrm{~K}$ for $1800 \mathrm{~s}$. The tensile strength after the tempering was $1586 \mathrm{MPa}$. The specimen surface for nanoindentation test was mechanically polished and then electropolished in a solution of $8 \%$ perchloric, $10 \%$ butylcellosolve, $60 \%$ ethanol and $22 \%$ water at $0{ }^{\circ} \mathrm{C}$ under 
the potential of 40 volts to remove the damaged layer. Nanoindentation experiments were performed using a Hysitron Triboindenter $\AA$; an atomic force microscope (AFM) mounted with a Triboscope ${ }^{\circledR}$ attachment providing both indentation and in-situ imaging capabilities. Probed sites and indent configurations on the specimen surfaces were confirmed before and after the indentation measurements. Smooth areas with surface roughness below $10 \mathrm{~nm}$ were chosen for indentation experiments. A Berkovich indenter was employed, and the tip truncation was calibrated by using a reference specimen of fused silica. Analyses for the tip calibration and the calculation of hardness were conducted by the method used by Oliver and Pharr [1]. Contacted peak loads, 100, 250, 500, 1000 and $2000 \mu \mathrm{N}$, were applied, respectively. Corresponding loading rates were $200 \mu \mathrm{N} / \mathrm{s}$ when the peak load was higher than $1000 \mu \mathrm{N}$, while the loading and unloading periods were set at a constant of $5 \mathrm{~s}$ when the peak load was lower than $1000 \mu \mathrm{N}$.

A tungsten single crystal was cut into pieces oriented with the (100), (110) and (111) surface. The samples were mechanically polished to a $0.3 \mu \mathrm{m}$ finish and then electropolished in a mixture of $10 \mathrm{~g} \mathrm{NaOH}, 100 \mathrm{ml} \mathrm{H}_{2} \mathrm{O}$ and $100 \mathrm{ml}$ glycerol at $40 \mathrm{~V}$ with a stainless steel cathode at $5^{\circ} \mathrm{C}$. The tungsten single crystal was chosen as a reference because it has a similar hardness to the tempered steel in addition to the uniform structure and isotropic elastic property [13]. In addition to the above mentioned five values, another peak load, $4000 \mu \mathrm{N}$, was also performed for the tungsten single crystal, in order to make the contact depth throughout cover the range of that for the steel specimen. It could be seen below. The same loading rates were used as the steel sample.

\section{RESULTS AND DISCUSSION}

Fig.1 shows a typical SEM micrograph of the tempered steel after the nanoindentation measurements of $8 \times 15$ pattern at an identical peak load of $500 \mu \mathrm{N}$. The contact depth was $30 \sim 40 \mathrm{~nm}$ for the indents. It should be remarked that the diameter of deformation zone is about 8 9 times of contact depth on the hemispherical approximation [14]. Therefore, the corresponding plastically deformed zone beneath the indenter expands to about $240 \sim 360 \mathrm{~nm}$ in diameter. The pitch of the indents is about $1 \mu \mathrm{m}$ in horizontal and $1.5 \mu \mathrm{m}$ in vertical. Consequently the deformed zone of the indention was not overlapped each other. As for cementite structure, it is seen in Fig.1 that cementite particles preferentially formed at block boundaries as well as prior austenite grain boundaries. It was found that the high hardness tends to be observed when indentation was conducted at positions near boundaries that were decorated by cementite particles, suggesting that the deviation of nanohardness is closely related to the density of cementite in the deformation zone.

When the applied peak load was changed from $100 \mu \mathrm{N}$ to $2000 \mu \mathrm{N}$, the contact depth varied from about $10 \mathrm{~nm}$ to100nm. Typical load-displacement curves for the tempered sample are shown in Fig.2. The curves are smooth and no displacement excursion is seen. The nanohardness of the tempered steel calculated using the method of Oliver and Pharr [1] are plotted as a function of contact depth in Fig.3. The results are an average of at
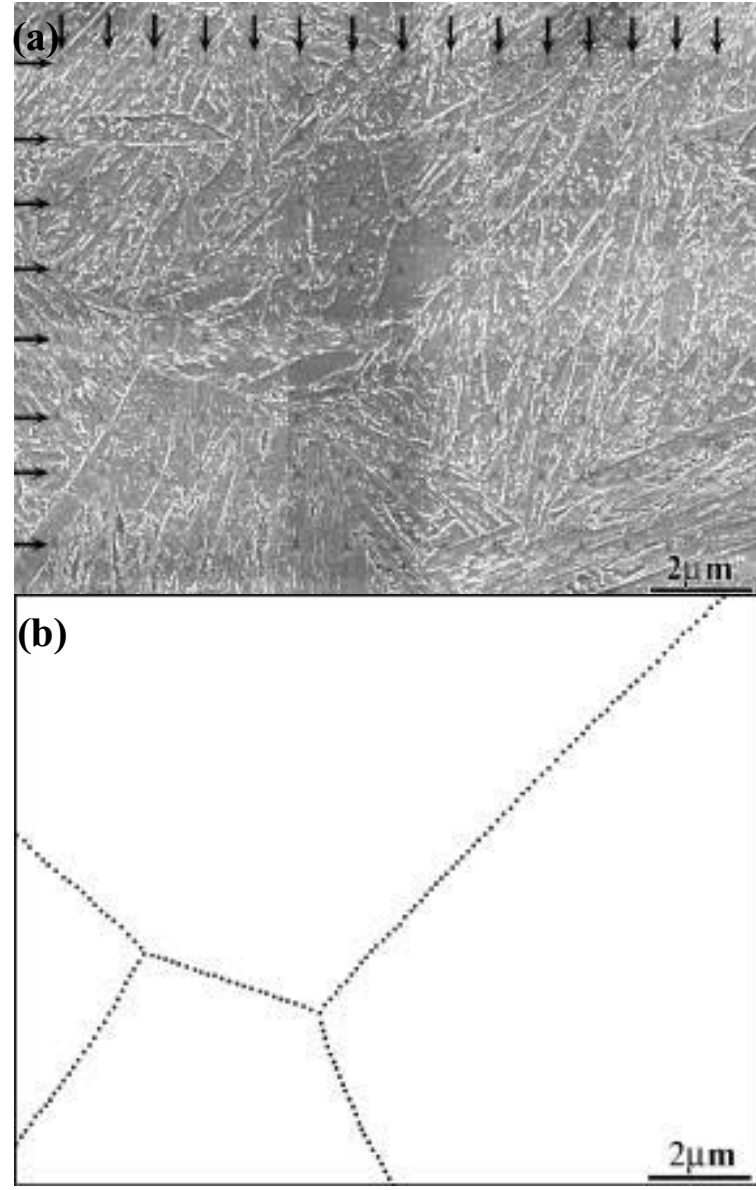

Fig.1 SEM image after the indentations with a peak load of $500 \mu \mathrm{N}$. Inner arrows indicate indentation lines and rows (a). The dots lines in (b) are the prior austenitic grain boundaries corresponding to the image (a).

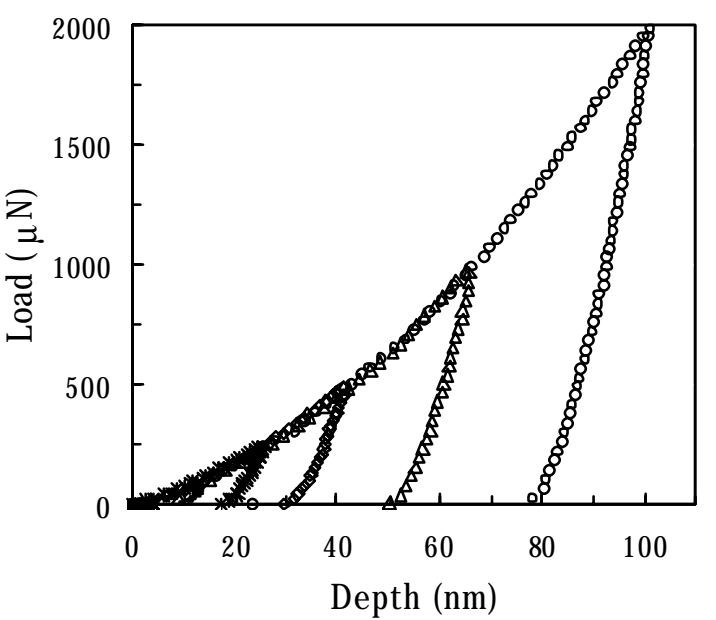

Fig.2 Load-displacement curves for the tempered steel.

least 50 indentations for each peak load with the error bars representing the standard deviation. It is easily seen that the hardness decreases quickly with increase in 


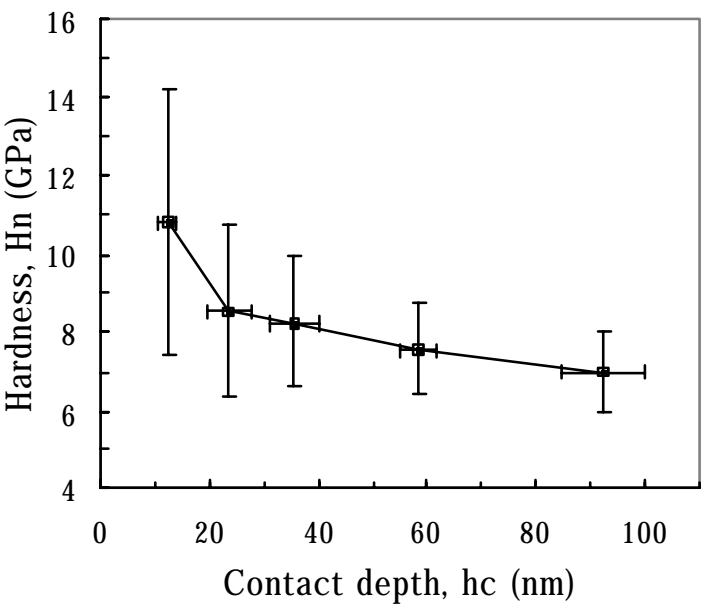

Fig.3 Hardness of the tempered steel as a function of the indentation depth. 50 to 150 indentations were performed. The error bar represents the standard deviation.

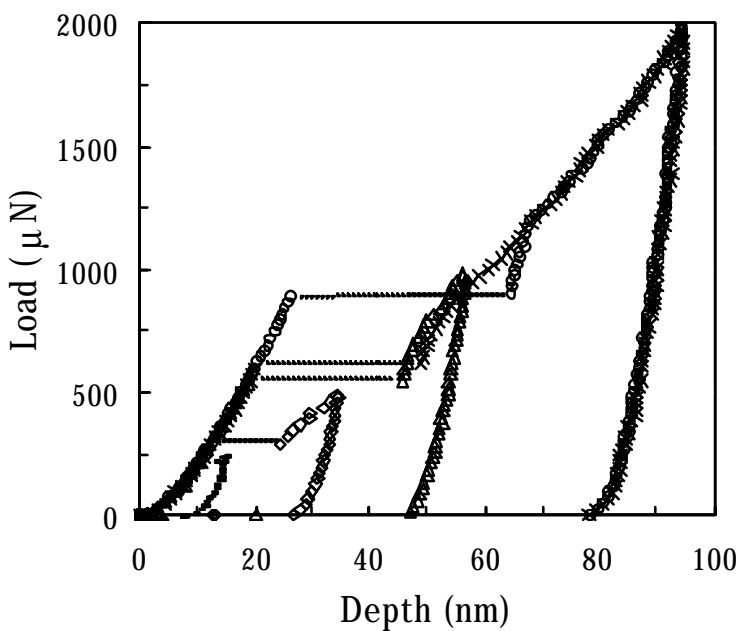

Fig.4 Load-depth curves of five indentations into the tungsten single crystal with (100) surface.

the indentation depth at extremely small penetration depths $($ hc $<25 \mathrm{~nm})$, and then decreases slowly within the measured range. The standard deviation is large at small penetration depths and tends to be a constant when the contact depth reaches $60 \mathrm{~nm}$ depth, corresponding the peak load of $1000 \mu \mathrm{N}$. It is interesting to note that the martensite block width of the same steel measured by Hayakawa et. al [15] was about $610 \mathrm{~nm}$, that is just larger than the diameter of deformation zone $(540 \mathrm{~nm}$; 9 times of $60 \mathrm{~nm}$ ) for $1000 \mu \mathrm{N}$ peak load. As described in Fig.1, cementite particles preferentially form at block boundaries. It is thus likely that the standard deviation of hardness becomes constant when the deformation zone becomes large enough to cover block boundaries. This means that the contact depth of $60 \mathrm{~nm}$ is the critical condition for disappearance of inhomogeneity effect on the nanohardness measurement for the present tempered steel. However, the standard deviation at the applied peak load of $1000 \mu \mathrm{N}$ is still large, $16.5 \%$ of its average hardness. The reasons for this large standard deviation will be discussed later.

Fig.4 illustrates typical load-depth curves of five indentations into an electropolished tungsten (100) crystal, in which pop-in behavior occurs. In fact most of the load-depth curves for tungsten (100) surface exhibit this characteristic. The abrupt bursts in displacement mean dislocation nucleation in the materials beneath the indenter [16], which implies that there is less internal stress and/or lower density of dislocation on the tested point. The tungsten single crystals with other two crystallographic orientations tested in this study also exhibited the abrupt bursts in displacement. It is noteworthy in Fig.4 that the load for the displacement excursion varies in a wide range from about $200 \mu \mathrm{N}$ to $900 \mu \mathrm{N}$. The reasons for such variations are attributed to oxide rupture and asperities of the sample surface [17].

Fig.5 represents the average of nanohardness as a function of contact depth with the error bars also reprensenting the standard deviation. 50 points were tested for each peak load. Even though the samples were electrically polished prior to nanoindentation testing and

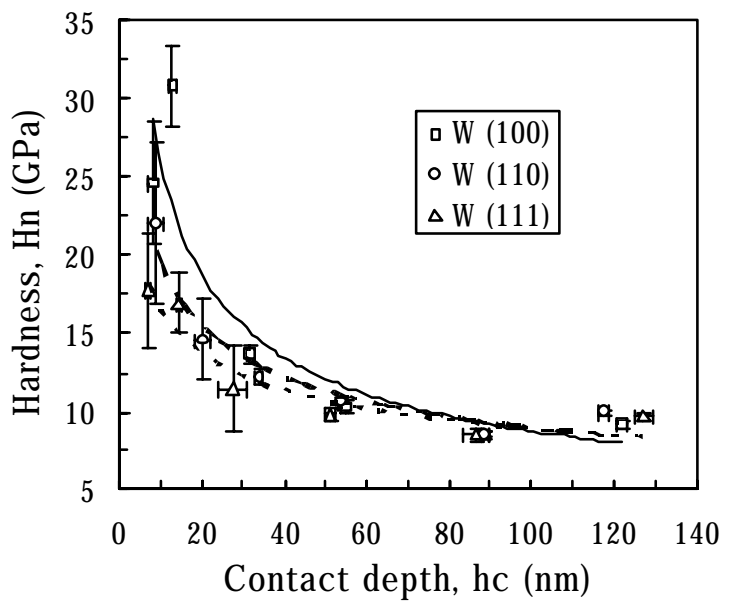

Fig.5 Relation between hardness and contact depth for the tungsten single crystals with (100), (110) and (111) surfaces. 50 indentations were performed for each crystal. The error bar represents the standard deviation.

smooth areas with surface roughness below $5 \mathrm{~nm}$ were chosen for indentation experiments, the measured hardness data at extremely shallow depths, hc $<20 \mathrm{~nm}$, exhibits a significant scatter for each oriented surface, and that the difference in hardness among them are evident. The (100) surface is the hardest one and (111) is the softest. In the range of contact depth from 20 to $55 \mathrm{~nm}$, the hardness continuously drops down for all the surfaces Further increasing the contact depth, the difference in hardness among them almost disappears. It means that the hardness value is not strongly related to the surface orientation when the contact depth is deep enough. This is due to that the tungsten exhibits very isotropic elastic property; its anisotropy ratio is 1.00 [13]. 
The distribution of hardness at $1000 \mu \mathrm{N}$ peak load for the tempered steel is shown in Fig.6. For comparison, the distributions of hardnesses of all the tested orientations for the tungsten single crystals, and the sum of all the

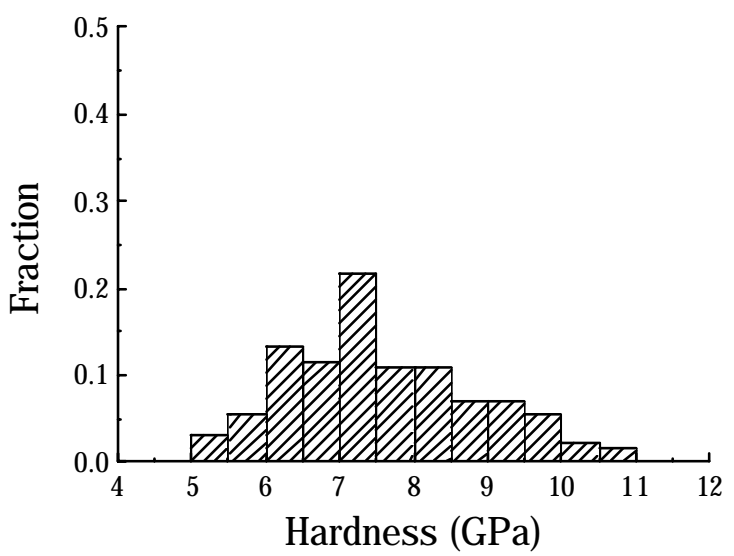

Fig.6 Distribution of hardness at a peak load of $1000 \mathrm{~N}$ for the tempered steel. three surfaces were shown in Fig.7. Once again, we selected the tungsten single crystal as a reference because its hardness is similar to that of the present tempered steel. Thus, it is reasonable to compare the deviation of the hardness between them. It is seen that the deviation of hardness of three tungsten surfaces is very narrower than that of the steel. At this peak load, the percentages of the standard deviation to their average value are $3.54 \%$, $3.46 \%$ and $4.48 \%$ for (100), (110) and (111) surface, and $4.88 \%$ for the sum of the three surfaces; whereas that of the steel is $16.5 \%$. The presence of surface atomic steps has a marked influence on the load-displacement response [17]. However if the sample surface is flat to the atomic degree, in principle, a single crystal may exhibit a small value for the deviation of hardness that is caused only by the test systematic error without the sample surface condition because the single crystal has a uniform structure and the same crystallographic orientation. This implies that the large deviation of the steel is resulted from not only the different crystallographic orientation of each tested point site, but also mainly the inhomogeneity of the multi-phase microstructure containing cementite particles, dislocations, and various kinds of boundaries.
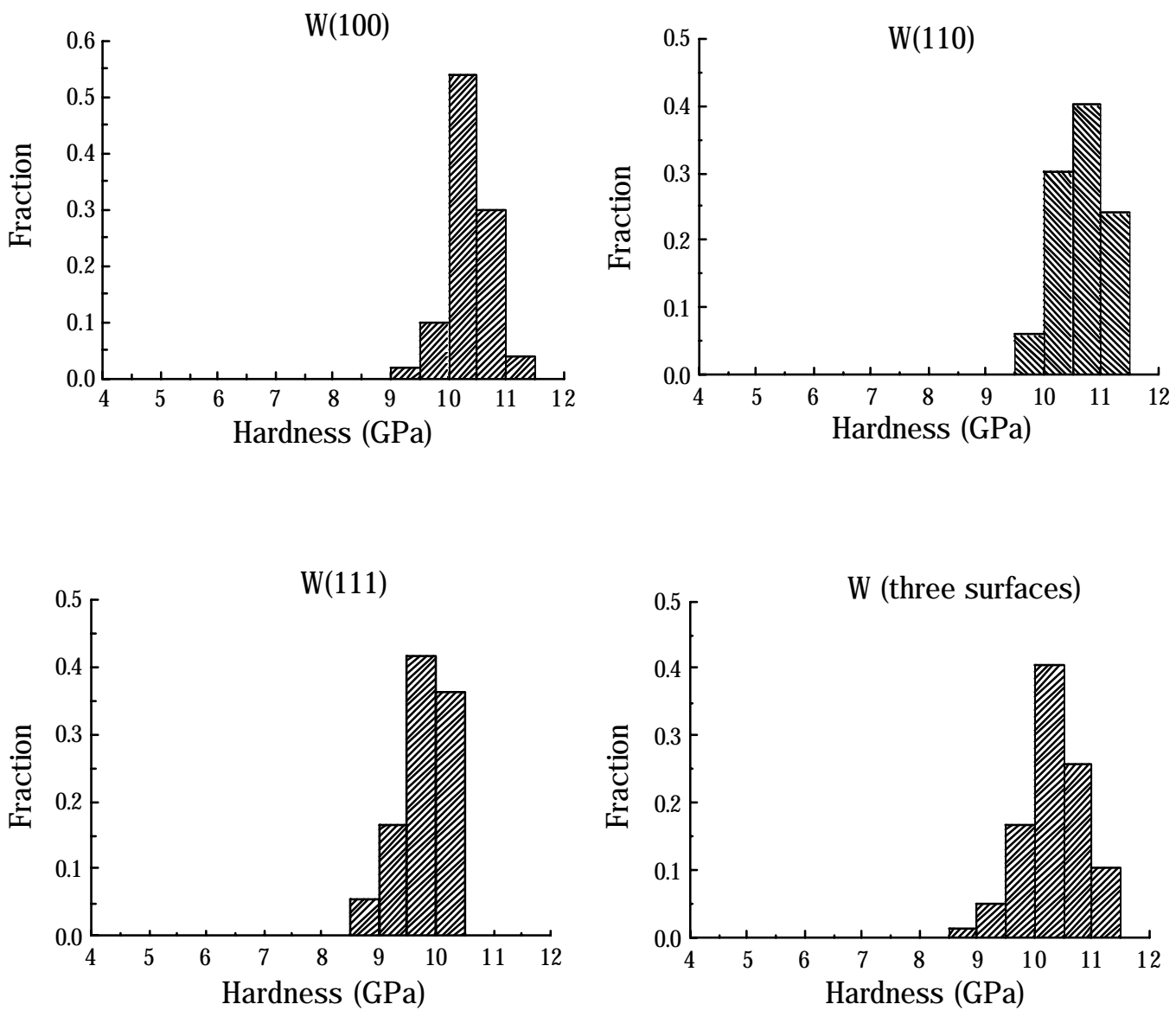

Fig.7 Distribution of hardness at a peak load of $1000 \mathrm{uN}$ for the tungsten single crystal with (100), (110) and (111) surfaces, and for all of the three surfaces. 


\section{CONCLUSIONS}

(1) The standard deviation of nanohardness decreases with increasing in the contact depth for all the tempered steel and the tungsten single crystals. As for the tempered steel, the standard deviation approaches a constant value when the contact depth reaches about $60 \mathrm{~nm}$ depth. Thus, $60 \mathrm{~nm}$ is considered to be the critical contact depth for the disappearance of inhomogeneity effect on the deformation behavior. The contact depth of $60 \mathrm{~nm}$ corresponds to the condition that the deformation zone covers block boundaries at which cementite particles preferentially form.

(2) The standard deviation of hardness at contact depths over $60 \mathrm{~nm}$ is much larger in the tempered steel than in the tungsten single crystals with (100), (110) and (111) surface. This large standard deviation for the tempered steel results from the inhomogeneity of the multi-phase microstructure containing cementite particles, dislocations, and various kinds of boundaries rather than different crystallographic orientation of each tested point site.

\section{ACKNOWLEDGMENTS}

One of the authors (Jinxu Li) expresses her hearty thanks to the Japan Society for the Promotion of Science for providing a JSPS Postdoctoral Fellowship from March 2002 to March 2004.

\section{REFERENCES}

1. W. C. Oliver and G. M. Pharr, J. mater. Res., 7(1992), pp. 1564-1583

2. S. Suresh and A. E. Giannakopoulos, Acta mater., 46
(1998), pp. 5755-5767

3. E. Giannakopoulos and S. Suresh, Scripta mater., 40 (1999), 1191-1198

4. Bolshakov, W. C. Oliver and G. M. Pharr, J. mater. Res., 11 (1996), 760-768

5. M. F. Doerner and W. D. Nix, J. mater. Res., 1 (1986), pp. 601-616

6. J. J. Vlassak and W. D. Nix, J. Mech. Phys. Solids, 42 (1994), pp. 1223-1245

7. J. B. Pethica, R. Hutchings and W. C. Oliver, Philos. Mag. A, 48 (1983), pp. 593-606

8. M. F. Doerner, D. S. Gardner and W. D. Nix, J. mater. Res., 1 (1986), pp. 845-851

9. M. J. Mayo, R. W. Siegel, Y. X. Liao and W. D. Nix, J. Mater. Res., 7 (1992), pp. 973-979

10. J.M. Marder, A.R. Marder Transactions of the ASM, Vol. 62, 1969, 1-10

11. A.R. Marder, G. Krauss, Transactions of the ASM, Vol.60, 1967, 651-660

12. A.R.Marder, G. Krauss, Transactions of the ASM, Vol. 62, 1969, 957-964

13. J. P. Hirth and J. Lothe, Theory of dislocations, $2^{\text {nd }}$ ed. John Wiley and Sons, New York (1982), pp. 837

14. Y. Murakami and M. Itokazu, Int. J. Solids Structures, 34 (1997), pp. 4005-4018

15. M. Hayakawa, T. Hara, S. Matsuoka and K. Tsuzaki, J. Japan Inst. Metals., 64 (2000), pp. 460-466

16. Gouldstone, H.-J. Koh, K.-Y. Zeng, A. E. Giannakopoulos and S. Suresh, Acta mater., 48 (2000), pp. 2277-2295

17. D. E. Kramer, K. B. Yoder and W. W. Gerberich, Phil. Mag. A, 81 (2001), pp. 2033-2058 